$\begin{array}{lllll}\text { September } & 13, & 2018 & 13: 56 & \text { WSPC/INSTRUCTION }\end{array}$ SFDMReview13 postproofs

FILE

Modern Physics Letters A

(C) World Scientific Publishing Company

\title{
COMPLEX SCALAR FIELD DARK MATTER ON GALACTIC SCALES
}

\author{
TANJA RINDLER-DALLER ${ }^{1,2}$ and PAUL R. SHAPIRO ${ }^{2}$ \\ ${ }^{1}$ Department of Physics and Michigan Center for Theoretical Physics, University of Michigan, \\ 450 Church Street, Ann Arbor, MI 48109, USA \\ ${ }^{2}$ Department of Astronomy and Texas Cosmology Center, The University of Texas at Austin, \\ 2515 Speedway C1400, Austin, TX 78712, USA
}

Received (Day Month Year)

Revised (Day Month Year)

\begin{abstract}
The nature of the cosmological dark matter remains elusive. Recent studies have advocated the possibility that dark matter could be composed of ultra-light, self-interacting bosons, forming a Bose-Einstein condensate in the very early Universe. We consider models which are charged under a global $U(1)$-symmetry such that the dark matter number is conserved. It can then be described as a classical complex scalar field which evolves in an expanding Universe. We present a brief review on the bounds on the model parameters from cosmological and galactic observations, along with the properties of galactic halos which result from such a dark matter candidate.
\end{abstract}

Keywords: cosmology; scalar-field dark matter; galactic halos.

PACS Nos.: 98.80.-k; 95.35.+d; 98.62.Hr; 14.80.-j

\section{Introduction and Motivation}

The nature of the cosmological dark matter is one of the most profound open questions in modern physics and cosmology. After many decades of research, it has become clear that dark matter underlies the formation of structure as we see it in the Universe today: galaxies and galaxy clusters reside in high-density filaments which surround voids where the density is comparatively low, giving rise to the large-scale cosmic web of structure. Numerical cosmological simulations have shown that largescale structure is best represented if the dark matter is assumed to be a collisionless and cold, i.e. non-relativistic, entity. On cosmological scales, therefore, it behaves like a 'dust-like' fluid.

Meanwhile, theories beyond the standard model (SM) of particle physics have been devised which are able to provide candidate particles for the dark matter (DM). The most popular and possibly best motivated candidates are the lightest supersymmetric particles in supersymmetric extensions of the SM. These are weaklyinteracting (i.e. subject to the weak force), massive particles (WIMPs). While mod- 
els allow generic values for their mass between around $1 \mathrm{GeV}$ to $10 \mathrm{TeV}$, direct and indirect detection experiments, including accelerator searches, are inconclusive and partly contradictory on their preferred exclusion limits. However, steady progress has been made in attempts to detect WIMP dark matter and it seems conceivable that it will be detected within the next decade, if it exists. However, in case detection experiments continue to deliver null-results, this may hint to an entirely different type of DM, e.g. the possibility that DM is a very low-energy phenomenon, behaving wave-like on macroscopic scales, instead of particle-like. Such a form of DM will be the topic of this review article.

In fact, one such DM candidate, equally prominent to WIMPs, has been the QCD axion, the pseudo Nambu-Goldstone boson (PNGB) that arises in the dynamical solution of the CP problem of the strong force (see Ref. 1 for a review). Indeed, with a mass of around $10^{-5} \mathrm{eV}$ and the fact that it is born non-thermally, the QCD axion can Bose-Einstein-condense, exhibiting coherence on the order of a de-Broglie wavelength of about $\lambda_{d e B} \sim 186 \mathrm{~m}$ in a galactic halo with a virial velocity of 200 $\mathrm{km} / \mathrm{s}$. However, this $\lambda_{d e B}$ is still a tiny number compared to galactic scales, and the QCD axion behaves like collisionless, cold dark matter (CDM) on all galactic and cosmological scales of interest in the present Universe. However, it has been pointed out in Ref. ${ }^{2}$ that the subsequent thermalization of the QCD axion to find a new ground state in the expanding background Universe may be 'memorized' such as to imprint different characteristics in the galactic dynamics, as compared to standard cold DM (CDM). This would be an interesting way of distinguishing WIMPs and axions by astronomical means, after all.

However, we will not consider the QCD axion in this review, but rather even much lighter particles, which are guaranteed to behave quantum-mechanically and hence distinctively from CDM on galactic scales. These DM candidates are motivated from a fundamental, as well as from an astrophysical point of view, as follows. In the very early Universe, PNGBs can arise generally when a global symmetry is spontaneously broken, while non-perturbative effects on lower energy scales break the symmetry explicitly, generating the (ultralow) mass. Examples include the aforementioned QCD axion, as well as familons, Majorons and related objects. Indeed, since theories beyond the standard model involve new symmetries, many of them global, which, upon breaking, will result in scalar and pseudoscalar PNGBs, the interest in those as dark matter candidates has lately seen a huge rise, see e.g. Ref. $\underline{3}$, Ref 4 and references therein. Ultralight scalar fields can also result as gravitational excitons in multidimensional cosmological models, giving rise to the dark matter in our observable Universe, see e.g. Ref. 5 . The choice of a potential in the Lagrangian which describes the scalar field will determine its cosmic evolution. We will be focusing on a scenario in which an ultra-light boson of mass around $10^{-21} \mathrm{eV} / c^{2}$ with a Higgs-like potential is responsible for all of the dark matter in the Universe. The boson shall be "charged" under a $U(1)$-symmetry, such that the dark matter number is conserved over the entire cosmic evolution, once it is in its condensed state, in which it will enter in the very early Universe. Its effective Langrangian shall thus 
be given by

$$
\mathcal{L}=\frac{\hbar^{2}}{2 m} g^{\mu \nu} \partial_{\mu} \psi^{*} \partial_{\nu} \psi-\frac{1}{2} m c^{2}|\psi|^{2}-\frac{g}{2}|\psi|^{4}
$$

(with metric signature $(+,-,-,-)$ ). The quartic term describes an effective 2boson self-interaction, which we choose to be non-negative, i.e. $g \geq 0$. As we will see, even tiny values for the self-interaction coupling strength $g$ can render those models surprisingly different from non-interacting ones. Equ.(11) assumes no effective coupling of the scalar field $\psi$ to SM particles, but only its minimal coupling to gravity

Scalar field dark matter (SFDM) provides a natural minimum scale for gravitational equilibrium, once perturbations grow nonlinear in the matter-dominated epoch. This makes it interesting for astrophysicists, who have found that the predictions of structure formation simulations using collisionless CDM are at odds with observations on small scales, especially at the level of dwarf and low surfacebrightness galaxies. Simulations predict cuspy galactic centers with DM densities going as $r^{-1}$ for $r \rightarrow 0$. In addition, many hundreds of subhalos are expected to surround host halos of Milky-Way size. On the other hand, observations tend to be better fit by cored profiles, and the number of observed satellite galaxies is smaller than predicted. These mismatches have been around for two decades and are known as the cusp/core and 'missing satellites' problems. In addition, it has been lately pointed out that the known classical dwarf spheroidal satellite galaxies of the Milky Way are not dense enough to populate the corresponding most massive subhalos found in simulations, a problem called 'too-big-to-fail'. It appears that all those problems could be cured if the DM densities in the innermost parts of galaxies were lower. While CDM-only simulations have their limitations and baryonic feedback processes have been suggested to reconcile observations with simulations, it remains a striking fact that it is just the DM-dominated galaxies which keep challenging the CDM predictions most (see Ref $\frac{10}{10}$ for a recent review on these problems). The above-mentioned minimum scale, therefore, is a welcoming feature of SFDM. That scale, however, is determined by the boson parameters, mass $m$ and self-interaction coupling strength $g$. So, astronomical observations can thereby help to establish or to rule out high-energy extensions of the SM.

In this brief review, we will almost exclusively restrict to results obtained for complex scalar fields with Lagrangian in Equ.(1), but refer interested readers to Ref. 11 for a review on real scalar fields and Ref. 12 for a wider application of complex scalar fields. Also, we will only consider works in which the scalar field itself constitutes the dark matter.

This article is organized as follows: in Section 2, we present basic equations and results which we will need for our discussion of galactic properties. Section 3

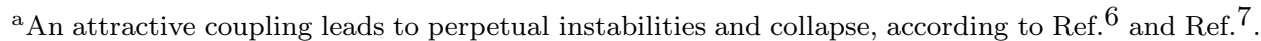
${ }^{\mathrm{b}}$ See also Ref ${ }^{[}$. On the other hand, Ref ${ }^{9}$ studies the implications of a coupling to baryons for the cosmic microwave background.
} 
September $\quad 13$ SFDMReview13 postproofs

summarizes some recent results which led to new bounds on the particle parameters $m$ and $g$. Finally, Section 4 will address the implications of SFDM in general and of these new bounds in particular on the structure of SFDM galactic halos.

\section{Basic equations for complex SFDM in the late Universe}

\subsection{Equations of motion}

SFDM obeys the Klein-Gordon equation in an expanding Universe. As soon as the rest-mass term dominates over other terms in the Langrangian (e.g. the quartic term in Equ.(10), once the density of SFDM drops significantly as a result of the expansion of the Universe, the (background) equation of state of SFDM will be dustlike. Since we assume that SFDM accounts for all of the dark matter, SFDM gives then rise to the epoch of matter-domination. We refer to this regime in the evolution of SFDM as Bose-Einstein condensed cold DM (BEC-CDM). In this epoch, it can then be treated in a Newtonian way, with the Klein-Gordon and Einstein equations of motion reducing to the non-linear Schrödinger equation and the Poisson equation. This holds true especially for the formation and evolution of galactic halos, whose dynamics involves non-relativistic velocities and gravitational fields. Thus, we will use the Newtonian framework of BEC-CDM in our description of galactic halos. Nevertheless, the quartic term in (11) is important in the early Universe and affects structure on small scales in the late Universe, i.e. in the matter-dominated epoch, as will be exemplified below.

The complex scalar field $\psi(\mathbf{r}, t)$ describing the ground state of SFDM in the matter-dominated era (BEC-CDM) satisfies the Schrödinger-Poisson (also called Gross-Pitaevskii-Poisson (GPP)) system of equations,

$$
\begin{gathered}
i \hbar \frac{\partial \psi}{\partial t}=-\frac{\hbar^{2}}{2 m} \Delta \psi+m \Phi \psi+g|\psi|^{2} \psi, \\
\Delta \Phi=4 \pi G m|\psi|^{2} .
\end{gathered}
$$

The number and mass density of DM in a given halo with gravitational potential $\Phi(\mathbf{r}, t)$ is then $n(\mathbf{r})=|\psi|^{2}(\mathbf{r})$ and $\rho(\mathbf{r})=m n(\mathbf{r})$, respectively.

The structure and evolution of BEC-CDM halos is governed by quantum-kinetic energy, gravity, and the self-interaction of identical dark matter bosons, as described by the quartic term in Equ.(11). The scattering cross section of indistinguishable bosons becomes constant in the low-energy limit,

$$
\sigma_{s}=8 \pi a_{s}^{2}
$$

with the s-wave scattering length $a_{s}$. The coupling constant of the effective interaction is then simply proportional to $a_{s}$,

$$
g=4 \pi \hbar^{2} a_{s} / m
$$

${ }^{\mathrm{c}}$ The only exception is the environment close to a central galactic black hole, which we will not consider. 
which is the (first) Born approximation.

It is remarkable that Equ.(2) lends itself to a hydrodynamic formulation, as originally observed by Ref.13, an attractive feature in the astrophysical context in which we use (2). Inserting the decomposition of the complex field into modulus and phase,

$$
\psi(\mathbf{r}, t)=|\psi|(\mathbf{r}, t) e^{i S(\mathbf{r}, t)}=\sqrt{\rho(\mathbf{r}, t) / m} e^{i S(\mathbf{r}, t)},
$$

into Equ.(2), leads to its splitting into a momentum and continuity equation, respectively,

$$
\rho \frac{\partial \mathbf{v}}{\partial t}+\rho(\mathbf{v} \cdot \nabla) \mathbf{v}=-\rho \nabla Q-\rho \nabla \Phi-\nabla P_{S I}
$$

and

$$
\frac{\partial \rho}{\partial t}+\nabla \cdot(\rho \mathbf{v})=0
$$

with the bulk velocity defined as $\mathbf{v}=\hbar \nabla S / m$. The more intuitive representation of those equations as hydrodynamic ones, however, comes at the expense of a more complicated, higher-order derivative in the first term of the rhs of (7). In fact, the gradient of

$$
Q=-\hbar^{2} \Delta \sqrt{\rho} /\left(2 m^{2} \sqrt{\rho}\right)
$$

gives rise to what is often called 'quantum pressure', an additional force on the rhs of Equ. (7), which basically stems from the quantum-mechanical uncertainty principle. It is responsible for the de Broglie length of the bosons as one characteristic length in the system: with the dimensions of $\Delta$ being $\mathrm{L}^{-2}$ and changing to the momentum representation, we see that

$$
L \sim \lambda_{d e B}=h / p=h /(m v) .
$$

On the other hand, the particle self-interaction results in a pressure of polytropic form in equ.(7),

$$
P_{S I}=K \rho^{2} \equiv g /\left(2 m^{2}\right) \rho^{2} .
$$

We will see that the corresponding length scale is proportional to $\sqrt{g / m^{2}}$ (see Equ.(19)).

The system of equations (2) and (3), or (7)-(8) with $\Delta \Phi=4 \pi G \rho$, respectively, will determine the properties of BEC-CDM structures which have decoupled from the Hubble expansion and undergo gravitational collapse.

\subsection{Gravitational equilibrium}

There is a notable body of literature on BEC-CDM halos in equilibrium and their properties, see e.g. Ref! $14|15 / 16 / 17| 18$ to name a few, with more references to follow below. 
The stationarity ansatz $\psi(\mathbf{r}, t)=\psi_{s}(\mathbf{r}) e^{-i \mu t / \hbar}$, where the GP chemical potential $\mu$ is fixed by the DM particle number, leads to the stationary form of equ.(2), or (7) 8), in which the mass density $\rho=m\left|\psi_{s}\right|^{2}$ and, hence, the gravitational potential $\Phi$ are time-independent. Such systems can be equivalently described by the GP energy functional, which is given by

$$
\mathcal{E}\left[\psi_{s}\right]=\int_{V}\left[\frac{\hbar^{2}}{2 m}\left|\nabla \psi_{s}\right|^{2}+\frac{m}{2} \Phi\left|\psi_{s}\right|^{2}+\frac{g}{2}\left|\psi_{s}\right|^{4}\right] d^{3} \mathbf{r} .
$$

Inserting again the decomposition $\psi_{s}(\mathbf{r})=\left|\psi_{s}\right|(\mathbf{r}) e^{i S_{s}(\mathbf{r})}$ into (12) (and omitting the subscript 's'), the total energy can be written as

$$
E=K+W+U_{S I},
$$

with the total kinetic energy

$$
K \equiv \int_{V} \frac{\hbar^{2}}{2 m}|\nabla \psi|^{2} d^{3} \mathbf{r}=\int_{V} \frac{\hbar^{2}}{2 m^{2}}(\nabla \sqrt{\rho})^{2} d^{3} \mathbf{r}+\int_{V} \frac{\rho}{2} \mathbf{v}^{2} d^{3} \mathbf{r},
$$

the gravitational potential energy

$$
W \equiv \int_{V} \rho \Phi / 2 d^{3} \mathbf{r}
$$

and the internal energy

$$
U_{S I} \equiv \int_{V} g \rho^{2} /\left(2 m^{2}\right) d^{3} \mathbf{r},
$$

which stems from the particle interactions, and which we have defined essentially as $U_{S I}=\int P_{S I} d V$ with $P_{S I}$ in (11).

These energy contributions enter the scalar virial theorem of an isolated BECCDM halo under self-gravity, which reads as

$$
2 K+W+3 U_{S I}=0 .
$$

As in classical gas dynamics, (17) (and possible boundary terms) can be derived by multiplying the equations of motion in fluid form, equ.(7), by $\mathbf{r}$ and integrating the resulting equation over volumes which enclose the system of interest. For an isolated body, a derivation involving a scaling argument was presented in Ref.19.

Now, the size of an object in hydrostatic equilibrium can be determined by solving Equ.(2)-(3), or (3) with Equ.(17)-(8). In fact, this has been done in Ref. ${ }^{20}$ in the limit where $g=0$, i.e. $P_{S I}=0$, and only quantum pressure will oppose gravity. The solution has no compact support, but the radius which includes 99 per cent of the mass reads

$$
R_{99}=9.9 \hbar^{2} /\left(G M m^{2}\right)
$$

It is easy to see that this is proportional to $\lambda_{d e B}$, Equ.(10), for a halo with corresponding virial velocity $v$. All structure below $R_{99}$ will be suppressed by means of the Heisenberg uncertainty principle. This regime has been called 'fuzzy DM' in Ref. 15 . Here, we will call this regime TYPE I BEC-CDM. On the other hand, in the 
opposite regime, when self-interaction is dominant, we can neglect $Q$ in Equ.(77). In that case, the equation of state is an $(n=1)$-polytrope with corresponding radius

$$
R_{0}=\pi \sqrt{\frac{g}{4 \pi G m^{2}}}
$$

(see also Ref. ${ }^{21 / 22}$ for complex SFDM and Ref. ${ }^{23 \mid 24}$ for real SFDM). We will call this regime TYPE II BEC-CDM. Here, $R_{0}$ is much larger than the corresponding value for $\lambda_{d e B}$, and, yet, it is the energy-independent cross section in the quantummechanical low-energy limit, Equ.(44), which provides that scale via (5) and (19). In either case, the central DM densities of the object turn out to be lower, as compared to CDM (see also Section 4.1). However, in both cases, the hydrostatic equilibrium size does not increase with the mass $M$ of the object, and hence we can not limit ourselves to either regime, in order to build up halos of variable size. The considerations in the next sections address attempts to overcome this problem, and in the course of that valuable constraints have been found.

\section{New bounds on the SFDM particle parameters}

\subsection{Boson scattering and relaxation times}

We have seen that the hydrostatic equilibrium size of TYPE II BEC-CDM halos is independent of halo mass, Equ. (19). Therefore, in order to build a hierarchy of halos which resembles observations, it is mandatory to go beyond the limitations of the pure TYPE II limit. The authors of Ref. ${ }^{25}$ have considered an interesting scenario in which a pure BEC-CDM halo core of polytrope radius (19) is enshrouded by an isothermal sphere of bosons in thermal equilibrium. This isothermal envelope serves as the halo outskirts and models flat galactic velocity profiles. In the process of detailing the features of this model, however, the authors can show that such a configuration must be ruled out, since the compliance to two critical observations leads to contradictory bounds on the boson parameters. As the authors of Ref. 25 show, reproducing realistic velocity profiles requires a smooth transition in the density between the polytropic core and the isothermal envelope, which can be recast in a lower bound on the boson mass. They find

$$
m \geq 10 \times\left(\frac{v_{c, \infty}}{100 \mathrm{~km} \mathrm{sec}^{-1}}\right)^{-1 / 4}\left(\frac{r_{c}}{1 \mathrm{kpc}}\right)^{-1 / 2} \mathrm{eV} / \mathrm{c}^{2},
$$

where $v_{c, \infty}$ is the asymptotic circular velocity of the isothermal envelope and $r_{c}$ is the minimum size of a halo core supported only by particle repulsion, i.e. it follows Equ.(19). Now, the authors also observe that, in the TYPE II regime, the DM scattering cross section per unit mass can be written as

$$
\sigma_{s} / m=8 G^{2} R_{0}^{4} m^{5} /\left(\pi^{3} \hbar^{4}\right),
$$

by combining Equ.(4), (5) and (19). Then, it is argued in Ref.25 that upper bounds on $\sigma / m$ for the elastic-scattering particles in the self-interacting DM model, i.e. 
CDM endowed with a finite cross section (referred to as "SIDM" in the literature), based upon comparing that model to astronomical observations, should apply to BEC-CDM, as weld. The interpretation of the Bullet cluster observations, for example, as a nearly collisionless merger of two cluster-sized halos has been found to limit $\sigma / m$ for SIDM halos to $(\sigma / m)_{\max }<1.25 \mathrm{~cm}^{2} / \mathrm{g}$, according to Ref.27, and this limit is imposed on Equ. (20) in Ref. ${ }^{25}$ in order to arrive at an upper bound on the boson mass of

$$
m<9.6 \times 10^{-4}\left(\frac{r_{c}}{1 \mathrm{kpc}}\right)^{-4 / 5} \mathrm{eV} / \mathrm{c}^{2}
$$

Obviously, these upper and lower bounds contradict each other for any realistic choice of halo parameters, and so the isothermal envelope can not make a cure to the radius-mass relationship of BEC-CDM halos. We can see that this conclusion is valid, even if we disregard the above SIDM limit from the Bullet cluster, as follows. The relaxation time for achieving thermodynamic equilibrium of a condensate fulfills to a good approximation the relationship, $\tau \simeq 1 /\left(\sqrt{2} n \sigma_{s} \bar{v}\right)$ with $n$ the condensate number density, $\sigma_{s}$ in Equ. (4) and $\bar{v}$ the mean value of the velocity distribution of the particles (see Ref. ${ }^{28}$ ), where we use the non-relativistic description, appropriate for the matter-dominated epoch, and sufficient for the sake of our estimate. Rewriting this formula in terms of $\bar{\rho} \sigma_{s} / m$, we can write the relaxation time of a spherical, uniform halo (core) as

$$
\begin{gathered}
\tau=1.889 \cdot 10^{111}\left(\frac{m}{m_{H}}\right)^{-5}\left(\frac{R}{1 \mathrm{kpc}}\right)^{-3 / 2}\left(\frac{M}{10^{8} M_{\odot}}\right)^{5 / 2} \times \\
\times\left(\frac{\bar{\rho}}{\mathrm{GeV} /\left(\mathrm{c}^{2} \mathrm{~cm}^{3}\right)}\right)^{-1}\left(\frac{\bar{v}}{100 \mathrm{~km} / \mathrm{sec}}\right)^{-1} \mathrm{sec},
\end{gathered}
$$

where we define the characteristic particle mass

$$
m_{H}=1.066 \cdot 10^{-22}\left(\frac{R}{1 \mathrm{kpc}}\right)^{-1 / 2}\left(\frac{M}{10^{8} M_{\odot}}\right)^{-1 / 2} \mathrm{eV} / \mathrm{c}^{2}
$$

(see Ref. ${ }^{29}$ ), and $\bar{\rho}$ denotes the mean halo density. Choosing the size of a typical dwarf spheroidal galaxy with $R=1 \mathrm{kpc}$ and $M=10^{8} M_{\odot}$, we see that $\tau$ will be larger than a Hubble time, $\tau \gtrsim 10^{17} \mathrm{sec}$, if

$$
m \leq 1.066 \cdot 10^{-3} \mathrm{eV} / \mathrm{c}^{2},
$$

in good agreement with the bound in Equ. (21) from Ref. ${ }^{25}$. Inserting those values into Equ. (20) results in the corresponding upper bound of

$$
\sigma_{s} / m \leq 2.1 \mathrm{~cm}^{2} / \mathrm{g},
$$

hence not so different from the bound in Ref. 27 . Thermodynamic equilibrium is thus not achieved for boson masses which obey inequality (24), i.e. for boson masses in

\footnotetext{
${ }^{\mathrm{d}}$ This reasoning has also been applied in Ref.26.
} 
which we are interestede. In Section 4.1, we will discuss a different scenario to overcome the problem of the mass-independent size.

\subsection{SFDM as an extra relativistic degree of freedom in the early Universe}

In the early Universe, the self-interaction term will dominate over the mass term in Equ.(1). It can be shown that the (background) equation of state of SFDM is then radiation-like. The timing of the transition from the radiation-like to the dust-like phase of SFDM must be in accordance with measurements of the redshift of matterradiation equality $z_{e q}$, as determined by the cosmic microwave background (CMB). This requirement has been noted before in Refs.21/23130, and further investigated in Ref. 31 . The requirement that SFDM has fully morphed into a CDM-like fluid by the time of $z_{e q}$ sets a constraint only on the ratio $g /\left(m c^{2}\right)^{2}$. In Ref $\frac{31}{\text {, we found }}$ that

$$
g /\left(m c^{2}\right)^{2} \leq 4 \times 10^{-17} \mathrm{eV}^{-1} \mathrm{~cm}^{3} .
$$

In addition, it has been found in Ref ${ }^{31}$ that, for complex SFDM, there is a transition at an even earlier epoch, when the kinetic term due to the phase in Equ.(1) takes over and the equation of state of complex SFDM changes from radiation-like to stiff-like, i.e. $\bar{p} \simeq \bar{\rho}$. Once in the stiff phase, the expansion rate of the universe is higher than in the radiation-dominated epoch, with $H \propto a^{-3}$, instead of $H \propto a^{-2}$. Hence, complex SFDM is the dominant cosmic component at these early epochs. In contrast to Ref. 30 , we do allow for SFDM-domination (in its stiff phase), prior to radiation-domination (see also Ref. ${ }^{32}$ ). The timely later transition from the stiff to the radiation-like phase of SFDM, which is equivalent to SFDM-domination giving way to radiation-domination, is constrained by the allowed amount of relativistic degrees of freedom $N_{\text {eff }}$ during Big Bang nucleosynthesis (BBN). Requiring that this transition is completed by the time of light nuclei production, we derive the following constraints on the boson parameters in Ref.31,

$$
m \geq 2.4 \times 10^{-21} \mathrm{eV} / \mathrm{c}^{2}
$$

and

$$
9.5 \times 10^{-19} \mathrm{eV}^{-1} \mathrm{~cm}^{3} \leq \mathrm{g} /\left(\mathrm{mc}^{2}\right)^{2} \leq 1.5 \times 10^{-16} \mathrm{eV}^{-1} \mathrm{~cm}^{3} .
$$

eWe note that these calculations differ from that in Ref.24, where a self-annihilating real scalar field is allowed to condense in the process of halo virialization, and a Bose-enhancement factor enters their expression for the relaxation time. This factor is only important at the BEC transition, but not far below it, see also Ref 28 . In the model we consider, however, condensation happened in the very early Universe. Also, we do not agree on the statement in Ref ${ }^{24}$ that it is impossible for complex SFDM to fulfill cosmological constraints, along with the required central density cusps of galaxies. In fact, density cusps are not supported in complex SFDM either, and so boson selfannihilation is not a prerequisite to explain different observations. See also Section 4.

${ }^{\mathrm{f}}$ This is the value which would make the equation of state parameter, $\langle\bar{w}\rangle \equiv\langle\bar{p}\rangle /\langle\bar{\rho}\rangle=0.001$. Note that Ref $\frac{31}{1}$ uses the notation $\lambda$ for the coupling strength $g$. 
To derive these bounds, we imposed the (conservative) constraint that the $N_{\text {eff }}$ during BBN be all the time within $1 \sigma$ of the measured value, $N_{\text {eff }}=3.71_{-0.45}^{+0.47}$, which we adopt from Ref 33 . Note that the bounds in Equ.(28) disfavor SFDM without self-interaction, i.e. fuzzy DM or TYPE I BEC-CDM ! Now, combining the bounds on $g /\left(m c^{2}\right)^{2}$ from equ.(26) and (28), results in corresponding bounds on the size of a virialized halo in TYPE II, by means of Equ.(19), according to

$$
0.75 \mathrm{kpc} \leq \mathrm{R}_{0} \leq 5.2 \mathrm{kpc} .
$$

As we pointed out in Ref 31 , it is a surprising and curious fact that the constraint on the number of relativistic degrees of freedom in the early Universe for the SFDM model leads to bounds on the size scale, which fit so well into the expected range of dwarf spheroidal and possibly smaller galaxies. By the same token, it is also clear that these bounds can shift upon future measurements using BBN and CMB.

In light of these new bounds on the particle parameters of SFDM (and necessarily BEC-CDM), as presented in this section, we will re-assess previous findings and study the implications of this dark matter model on halo properties in the next section.

\section{Implications for galactic structure}

\subsection{A hierarchy of BEC-CDM halos}

The characteristic length scales due to the quantum nature of BEC-CDM result in the suppression of the formation of objects below those scales, while structure formation on larger scales is expected to follow the lore of standard CDM. Indeed, the power spectrum of linear DM perturbations resembles the one for $\Lambda$ CDM, except for the fact that the turnover happens at lower wavenumbers and perturbations beyond a certain cutoff are suppressed, by analogy with neutrinos (hot DM) or sterile neutrinos (warm DM). This cutoff necessarily depends on the values of the SFDM particle parameters, see Ref. ${ }^{[5]}$ and Ref. ${ }^{34}$. Some model parameters, for instance a real field with the popular value of $m=10^{-23} \mathrm{eV} / c^{2}$ and no self-interaction, i.e. fuzzy DM, are claimed to pass the test in the sense of resembling $\Lambda \mathrm{CDM}$ on large scales, while appropriately suppressing structure on small scales. On the other hand, we have seen above that models of complex SFDM without self-interaction are disfavored for the current number of $N_{\text {eff }}$ during BBN. Also, the lower bound on particle mass derived in Ref. ${ }^{31}$, Eq.(27), excludes masses as low as $10^{-23} \mathrm{eV} / c^{2}$. A much more detailed exploration of the parameter space of SFDM models is necessary to draw further conclusions. The study of the linear growth of structure in a Universe with complex SFDM is more complicated because of the additional degree of freedom due to the phase of the complex field. Anisotropies in the corresponding perturbed energy-momentum tensor have to be considered carefully, along with non-trivial boundary and initial conditions. These issues are especially critical before the matter-dominated epoch. A detailed analysis of perturbations in the regime of matter-domination, however, has been presented in Ref. $[35$ and Ref. 
The results of these studies basically confirm that SFDM of the form of Equ.(7) with (11) has a growing mode solution for the linear overdensity as for cold dust, i.e. $\delta \equiv \delta \rho / \bar{\rho} \propto a$, with the scale factor $a$.

However, for lack of cosmological simulations, it shall be sufficient here to outline the envisaged picture in an analytic manner, continuing along the line of Ref.29, by using a simple top-hat collapse scenario (see also Ref ${ }^{37}$ for SFDM without selfinteraction). A numerical infall study with cosmological boundary conditions will be presented elsewhere. We have noted in Section 2.2 that the equilibrium size $R$ of virialized BEC-CDM halos of mass $M$ is in conflict with observations: for a given set of particle parameters, the product $R M=$ const. for TYPE I BEC-CDM, while $R$ is a function of density (but independent of $M$ ) for TYPE II BEC-CDM, see Equs. (18) and (19). In conjunction with the disfavoring of TYPE I according to Ref. 31 , this suggests that it is desirable to improve upon models of TYPE II. The attempt to enshroud a TYPE II halo core with an isothermal sphere of thermalised bosons to overcome the size problem has been shown in Ref. ${ }^{25}$ to lead to contradictions, ruling out this scenario (see Section 3.1). In Ref. 29 , on the other hand, we have argued that it is the kinetic energy of (possibly) coherent wave motion, which may help to grow halos of the size range observed. Numerical studies of the virialization process of isolated, self-gravitating BEC blobs upon collapse show oscillations and mass ejection away from the 'to-be' virialized core, termed "gravitational cooling" in Ref. 38 in the context of the related phenomenon of boson stars. This feature is independent of the particle masses chosen, as long as the system itself can be described by a Schrödinger equation, see Ref. $39|40| 41$. It is therefore conceivable to envisage a picture where it is only the minimum size halos (or halo cores in larger galaxies) which obey the virial equilibrium of TYPE II BEC-CDM in the form of Equ.(19), while larger halos have to have additional energy contributions due to wave motion and possibly boundary terms in the virial theorem.

Consider a 'classic' top-hat collapse scenario in an Einstein-de-Sitter universe for BEC-CDM without kinetic energy to derive the minimum size for BEC-CDM halos. We denote its quantities with subscript 'zero'. A density perturbation is considered to decouple from the general Hubble expansion at the turn-around radius $r_{t a, 0}$. There, we require that the total energy is entirely gravitationally, i.e. $E_{t a, 0}=W_{t a, 0}$, and $U_{t a, 0}=0, K_{t a, 0}=0$, see Equ.(13). While $U_{t a, 0}$ cannot completely vanish in reality due to the finite (albeit, small) self-interaction, neglecting its contribution is basically identical to the requirement and expectation that BEC-CDM behaves dust-like, i.e. like collisionless CDM, at the time of infall, prior to virialization. According to the standard uniform sphere approximation for the post-collapse, virialized object that results from top-hat collapse, we assume the post-collapse sphere has uniform density $\rho_{0}$, which fulfills virial equilibrium, Equ.(17) with $K=0$, re- 
sulting in a corresponding virial radius $\mathrm{g}$ of

$$
R_{T H, 0}=\sqrt{\frac{15}{2}}\left(\frac{g}{4 \pi G m^{2}}\right)^{1 / 2} .
$$

It is easy to show that the collapse ratios are given by

$$
R_{T H, 0} / r_{t a, 0}=2 / 3 \text { and } \rho_{0} / \rho_{t a, 0}=(3 / 2)^{3}
$$

(see Ref. ${ }^{29}$ ), where $r_{t a, 0}$ and $\rho_{t a, 0}$ are the radius and density of the pre-collapse sphere. Requiring in addition that the top-hat density is proportional to the background density at the time of collapse $\rho_{b, \text { coll }}$, we can see that

$$
\rho_{0}=\left(\frac{3}{2}\right)^{3} \rho_{t a, 0}=\left(\frac{3}{2}\right)^{3} \frac{9 \pi^{2}}{4} \rho_{b, \text { coll }}=\frac{243 \pi^{2}}{32} \rho_{b, \text { coll }} \simeq 75 \rho_{b, \text { coll }} .
$$

Note that this factor is significantly smaller than the standard value of $18 \pi^{2} \simeq 178$ for collisionless CDM. Thus, already at this simple level is it evident that BEC-CDM will result in collapsed structures of lower density h. However, $R_{T H, 0}$ and $r_{t a, 0}$ have a unique value, once the ratio of particle parameters $\mathrm{g} / \mathrm{m}^{2}$ is fixed. In practice, we will want to fix the minimum size $R_{T H, 0}$, motivated by galaxy observations, to determine the allowed value of particle parameters. The exact value of $R_{T H, 0}$, however, is not necessary in outlining the general idea.

Now, in order to build halos of size $R$ larger than $R_{T H, 0}$, we include an (effective) kinetic term $K_{\text {eff }}$ in the virial theorem (17), which shall capture the overall kinetic energy due to wave motions. For the general argument outlined here, it is sufficient to consider a non-vanishing bulk velocity $\mathbf{v}$, such that $K_{\text {eff }}=\int \frac{\rho}{2} \mathbf{v}^{2} d V>0$. Since we restrict our analysis to top-hats with uniform density $\rho$, we may consider the gross average of $K_{\text {eff }}=\frac{3}{2} M \sigma_{v}^{2}$ with the top-hat mass $M$ and velocity dispersion $\sigma_{v}^{2}=\frac{1}{3}\left\langle\mathbf{v}^{2}\right\rangle$. Using (17) with that form of $K_{\mathrm{eff}}$, we can write the velocity dispersion as

$$
\sigma_{v}^{2}=G M /(5 R)-K \rho=G M /(5 R)\left[1-\left(R_{T H, 0} / R\right)\right]^{2},
$$

or in fiducial units (after taking the root)

$$
\sigma_{v}=9.275\left[1-\left(\frac{R_{T H, 0}}{R}\right)^{2}\right]^{1 / 2}\left(\frac{M}{10^{8} M_{\odot}}\right)^{1 / 2}\left(\frac{1 \mathrm{kpc}}{R}\right)^{1 / 2} \mathrm{~km} / \mathrm{sec} .
$$

Additionally, in order for the virial radius $R$ to depend on halo mass and redshift of collapse $z_{\text {coll }}$, as they do for standard CDM, we require the top-hat density to be a fixed fraction of the background density at $z_{\text {coll }}$, that is, $\rho=C \rho_{b, c o l l}$, where the

${ }^{g}$ The different prefactor compared to the one in Equ. (19) stems from the fact that the density is uniform, instead of following an $(n=1)$-polytropic run.

${ }^{\mathrm{h}}$ This suggests that a too literal adoption of the standard CDM framework for halo mass functions and profiles to dark matter models with a significant fraction of ultra-light axions can be problematic, see Ref. 12 . It is very interesting, though, that the analysis of these authors also hint to a mass of $m \sim 10^{-21} \mathrm{eV} / c^{2}$, close to the bound in Equ. (27), by using entirely different constraints. 
factor $C$ has to be determined numerically using Equ.(37) below. The corresponding total mass is then $M=4 \pi R^{3} C \rho_{b, c o l l} / 3$, which yields a radius

$$
R\left(M, z_{\text {coll }}\right)=\left[3 M /\left(4 \pi C \rho_{b, \text { coll }}\right)\right]^{1 / 3} .
$$

Repeating the above calculation for these larger top-hats with $E_{t a}=W_{t a}$ and $E_{\text {post-collapse }}=W+U+K_{\text {eff }}$, we obtain for the corresponding collapse ratio

$$
\frac{R}{r_{t a}}=1-\frac{\sigma_{v}^{2}}{2\left(\sigma_{v}^{2}+K \rho\right)}-\frac{K \rho}{3\left(\sigma_{v}^{2}+K \rho\right)}=\frac{\sigma_{v}^{2} / 2+2 K \rho / 3}{\sigma_{v}^{2}+K \rho} .
$$

The corresponding generalization of Equ.(32) is then given by

$$
\rho=\frac{9 \pi^{2}}{4} \rho_{b, \text { coll }}\left(\frac{\sigma_{v}^{2}+K \rho}{\sigma_{v}^{2} / 2+2 K \rho / 3}\right)^{3}
$$

again assuming an EdS universe with dust in order to relate $\rho_{t a}=9 \pi^{2} \rho_{b, \text { coll }} / 4$. However, note that Equ.(36) and (37) are not explicit and must be solved numerically for any given $K$ and non-vanishing $\sigma_{v}$. The standard CDM results of $\sigma_{v}=\sqrt{G M /(5 R)}$, $R / r_{t a}=1 / 2$ and $\rho / \rho_{b, \text { coll }}=18 \pi^{2}$ are recovered if $g / m^{2} \equiv 0$, since then $K=0$ and hence $R_{T H, 0}=0$. So, we see that the collapse ratios $R / r_{t a}$ and $\rho / \rho_{b, c o l l}$ are not universal due to the lower size cutoff of DM perturbations, provided by BEC-CDM. The resulting smallest halos have a size of $R_{T H, 0}$, a mass of $M_{\min }=4 \pi \rho_{0} R_{T H, 0}^{3} / 3$ and $\sigma_{v}=0$ by construction. On the other hand, larger halos will follow the relationship (35) with a non-vanishing velocity dispersion due to internal wave motion, according to (34). This guarantees that BEC-CDM halos of mass $M>M_{\min }$ would share the mass-radius relation of halos in the standard CDM model, if halos of a given mass $M$ typically collapse at the same time as they do for standard CDM. The standard CDM relationships will be more and more accurate the higher the velocity dispersion of the DM halo becomes, i.e. the larger $R / R_{T H, 0}$. Therefore, the clustering and halo properties on scales beyond the smallest galaxies and halo cores, respectively, will be more or less the same for BEC-CDM as for collisionless CDM.

The particle parameters, which enter $R_{T H, 0}$ in the combination $\mathrm{g} / \mathrm{m}^{2}$ may now be chosen such that $M_{\min }$ corresponds to the halo mass of the smallest observed galaxies, as well as to the DM core mass of large galaxies. To address the cusp-core issue, along with the "missing satellite" problem, we may choose a fiducial value of $R_{T H, 0}=1 \mathrm{kpc}$, the order of magnitude at which these problems arise, and which is in accordance with the bounds in Equ.(29). Also, observations of Milky Way dwarf spheroidal galaxies suggest that they host about $10^{7} M_{\odot}$ within the central $300 \mathrm{pc}$ Ref. $\frac{43}{4},(2-7) \times 10^{7} M_{\odot}$ within about 600 pc Ref. 4 , and as a result virial masses of $10^{8}-10^{9} M_{\odot}$ with maximum velocity dispersions of order $\sigma_{v} \simeq 10 \mathrm{~km} / \mathrm{sec}$. Thus, a fiducial choice of $R_{T H, 0}=1 \mathrm{kpc}$ and $M_{\min }=10^{8} M_{\odot}$ seems reasonable. 
$\begin{array}{lllll}\text { September } & 13, & 2018 & 13: 56 & \text { WSPC/INSTRUCTION }\end{array}$ SFDMReview13 postproofs

\subsection{Signature effects unique to BEC-CDM halos}

\subsubsection{Halo shapes and quantized vortices}

The standard scenario of structure formation expects that halos will acquire angular momentum in the course of tidal-torquing due to the surrounding large-scale structure. Galaxies are observed to have angular momentum, which must have been seeded by the DM in whose potential wells they formed. N-body simulations of cosmic DM structure formation do indeed confirm the basic expectations from tidaltorque theory, confirming non-spherical shapes, even though the amount of angular momentum is small, and DM halos are far from being rotationally supported. Values for the dimensionless spin parameter $\lambda \equiv L|E|^{1 / 2} /\left(G M^{5 / 2}\right)$, with $L$ the total angular momentum, and $E$ the total energy of a halo of mass $M$, cover a typical range of about $0.01-0.1$ with median values of about $0.03-0.05$, for halos in the

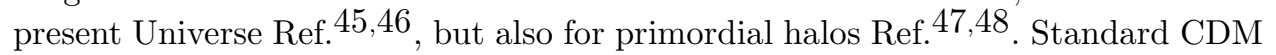
also seems to prefer a distribution of shapes, with oblate and prolate axis ratios for higher and highest mass halos. The study of halo shapes in BEC-CDM, on the other hand, has hardly been considered and results are very scarce. In Ref. $\frac{18}{1}$, we have studied some simple, but analytic models. BEC-CDM halo velocity fields obey the irrotationality condition for angular momenta which are lower than the threshold for forming a (singly-quantized) vortex, which breaks this condition locally. For an axisymmetric vortex in the center of a halo, this threshold is given by $L_{Q M} \equiv \frac{M}{m} \hbar$, which, for a given $\lambda$ and $L$ becomes a minimum condition on the particle mass. Rotation of the halo imprints a non-vanishing phase gradient in the scalar field, and we expect non-spherical shapes. In Ref.18, we have shown that the irrotationality condition will force TYPE II BEC-CDM halos to be of prolate form. More precisely, it can be shown that halos can be described by Riemann-S ellipsoids before and after vortex formation. Recently, the authors of Ref $\frac{49}{4}$ conclude from numerical tests that the inclusion of angular momentum may be a way to make rotation curves of BEC-CDM halos fit better to observations, at least as long as no vortices arise.

The formation of quantized vortices in SFDM has attracted comparatively more attention. In Ref. 50 , it was argued that, if DM is composed of ultra-light BEC$\mathrm{CDM}$, the rotation velocity of the Andromeda galaxy would be sufficient to create vortices. Subsequently, it was shown in Ref $[51$ that a lattice of about 500 vortices can produce a velocity profile which can fit data for the Milky Way. Recently, in Ref $[52$ the detailed density profile of a spherical halo in the presence of a central vortex was calculated, and limits on the boson parameters for a vortex to form in the Andromeda galaxy were derived. All those works, however, were limited to spherical halo shapes. On the other hand, in Ref ${ }^{18}$ we have derived the bounds on the boson parameters for vortex formation, resulting from self-consistent Maclaurin and Riemann-S ellipsoidal solutions of the GPP system, which have the same values for the spin parameter $\lambda$, as we expect from standard CDM. Vortex formation requires a minimum mass $m \geq m_{\text {crit }}$ and a minimum coupling strength $g \geq g_{\text {crit }}$. These critical values are smaller for larger $\lambda$, from which larger vortex cores result. 
According to Table II and III of Ref. ${ }^{29}$ for a fiducial halo of dwarf spheroidal size of $R=1 \mathrm{kpc}$, the critical values for the particle parameters above which vortex formation happens are lower than the bound in Equ.(27) and (28) if $\lambda \geq 0.05$ or $\lambda \geq 0.1$, respectively, depending on the halo model. That is, vortex formation can be avoided only if $\lambda \leq 0.05$. This is an interesting result, since it can imply a depleted DM density in the centers of such galaxies due to a central vortex. More detailed modeling along with a careful comparison to observed velocity profiles of dwarf spheroidals will be an important additional test-bed for SFDM.

\subsubsection{Halo mergers}

On the small scales on which BEC-CDM differs from CDM, as described above, the wave coherence of BEC-CDM can result in distinctive features upon halo collision and merging. Indeed, for different potentials of real scalar fields, it has been

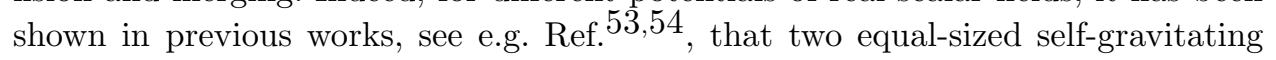
BEC-CDM blobs will be colliding and merging to form a single structure if the total energy of the system is negative. The relaxation process results again in the ejection of scalar field, i.e. gravitational cooling. However, if the total energy of the system is positive, the two blobs can pass through each other intactly, i.e. they can exhibit solitonic behavior. While previous studies have established the generality of these phenomena, we still lack a detailed modeling of halo mergers and comparison to observations (for instance to the Bullet galaxy cluster), which would result in particle parameter space exclusion regions. It remains to be seen whether the allowed parameter space of SFDM from cosmological and galactic observations may be challengend further by the distinctive phenomenology of halo mergers. Since BEC-CDM halos become more and more CDM-like at higher masses, we would not expect dramatic differences on the scales of galaxy cluster collisions, except for the very cluster centers of order $1 \mathrm{kpc}$. As far as observations can identify the degree of collisionality on those scales, whether for clusters or galaxies, this will enable to constrain SFDM (and hence BEC-CDM) further. However, cosmological simulations of SFDM are needed to answer those questions in more detail. In fact, the work of Ref. 55 remains the only realistic structure formation simulation of complex SFDM, but without self-interaction (i.e. fuzzy DM). Indeed, a few halo mergers with rather complicated interference patters are observed within a simulation volume of side length $1 \mathrm{Mpc} / \mathrm{h}$. However, one unexpected outcome consists of cusps in the density profiles of those halos. This is very counter-intuitive, given the analytial expectation that cusps are prohibited due to the inherent characteristic length scales of BEC$\mathrm{CDM}$, and is also in contrast to numerical results of Ref.15 in 1D. We believe that the use of the pseudo-spectral method in solving the Schrödinger equation in Ref. could be too insensitive to capture the detailed shock physics at halo formation and merging, and may lead to poor resolutions of the halo centers, after all.

This result along with the other ones we have been trying to summarize in this review will hopefully spur on more activity in complex scalar field dark matter, the 
September $\quad 13, \quad 2018 \quad 13: 56 \quad$ WSPC/INSTRUCTION $\quad$ FILE SFDMReview13 postproofs

interesting alternative to collisionless CDM and the WIMP paradigm.

\section{Acknowledgments}

This work was supported in part by U.S. NSF grants AST-0708176, AST-1009799 and NASA grants NNX07AH09G, NNG04G177G, NNX11AE09G to PRS. TRD also acknowledges support by the Texas Cosmology Center of the University of Texas at Austin and by the Michigan Center for Theoretical Physics of the University of Michigan.

\section{References}

1. R.D. Peccei, in Lect.Notes Phys. 741, 3-17, Springer, 2008

2. P.Sikivie, Q.Yang, Phys.Rev.Lett. 103, 111301 (2009)

3. J.A. Frieman, C.T. Hill, A. Stebbins, I. Waga, Phys.Rev.Lett. 75, 2077 (1995)

4. A. Arvanitaki, S. Dimopoulos, S. Dubovsky, N. Kaloper, J. March-Russell, Phys.Rev. D 81, $123530(2010)$

5. U. Günther, A. Zhuk, Phys.Rev. D 56, 6391 (1997)

6. T. Fukuyama, M. Morikawa, T. Tatekawa, J. Cosmol. Astropart. Phys. 06, 033 (2008)

7. T. Fukuyama, M. Morikawa, Phys.Rev. D 80, 063520 (2009)

8. S.M. Carroll, Phys.Rev.Lett. 81, 3067 (1998)

9. F. Ferrer, J.A.Grifols, J. Cosmol. Astropart. Phys. 12, 012 (2004)

10. D.H. Weinberg, J.S. Bullock, F. Governato, R. Kuzio de Naray, A.H.G. Peter, arXiv:1306.0913, (2013)

11. J. Magaña, T. Matos, J.Phys.:Conf.Series 378, 012012 (2012)

12. F.S. Guzmán, J.Phys.:Conf.Series 91, 012003 (2007)

13. E. Madelung, Z.für Phys. 40, 322 (1927)

14. S.J. Sin, Phys.Rev. D 50, 3650 (1994)

15. W. Hu, R. Barkana, A. Gruzinov, Phys.Rev.Lett. 85, 1158 (2000)

16. M. Alcubierre, F.S. Guzmán, T. Matos, D. Núñez, L.A. Ureña-López, P. Wiederhold, Class.Quant.Grav. 19, 5017 (2002)

17. J.-W. Lee, S. Lim, J. Cosmol. Astropart. Phys. 7, 01 (2010)

18. T. Rindler-Daller, P.R. Shapiro, Mon. Not. R. Astron. Soc. 422, 135 (2012)

19. X.Z. Wang, Phys.Rev. D 64, 124009 (2001)

20. M. Membrado, A.F. Pacheco, J. Sañudo, Phys.Rev.A 39, 4207 (1989)

21. J. Goodman, New Astronomy 5, no.2, 103 (2000)

22. C.G. Böhmer, T. Harko, J. Cosmol. Astropart. Phys. 06, 025 (2007)

23. P.J.E. Peebles, Ap. J. 534, L127 (2000)

24. A. Riotto, I. Tkachev, Phys.Lett. B 484, 177 (2000)

25. Z. Slepian, J. Goodman, Mon. Not. R. Astron. Soc. 427, 839 (2012)

26. T. Matos, L.A. Ureña-López, Phys.Lett. B 538, 246 (2002)

27. S.W. Randall, M. Markevitch, D. Clowe, A.H. Gonzalez, M. Bradač, Ap. J. 679, 1173 (2008)

28. A. Griffin, T. Nikuni, E. Zaremba, Bose-condensed gases at finite temperatures, Cambridge Univ.Press (2009)

29. T. Rindler-Daller, P.R. Shapiro, Astrophys. Space Sci. Proc. 38 (2013), in press, arXiv:1209.1835

30. A. Arbey, J. Lesgourgues, P. Salati, Phys.Rev. D 65, 083514 (2002)

31. B. Li, T. Rindler-Daller, P.R. Shapiro, arXiv:1310.6061 (2013) 
32. A. Arbey, Phys.Rev. D 74, 043516 (2006)

33. G. Steigman, arXiv:1208.0032 (2012)

34. T. Matos, L.A. Ureña-López, Phys.Rev. D 63, 063506 (2001)

35. T. Harko, Mon. Not. R. Astron. Soc. 413, 3095 (2011)

36. P.H. Chavanis, Astron. Astrophys. 537, A127 (2012)

37. F.S. Guzmán, L.A. Ureña-López, Phys.Rev. D 68, 024023 (2003)

38. E. Seidel, W.-M. Suen, Phys.Rev.Lett. 72, 2516 (1994)

39. E. Seidel, W.-M. Suen, Phys.Rev. D 42, 384 (1990)

40. J. Balakrishna, E. Seidel, W.-M. Suen, Phys.Rev. D 58, 104004 (1998)

41. F.S. Guzmán, L.A. Ureña-López, Phys.Rev. D 69, 124033 (2004)

42. D.J.E. Marsh, J. Silk, Mon. Not. R. Astron. Soc. 437, 2652 (2014)

43. L.E. Strigari, J.S. Bullock, M. Kaplinghat, J.D. Simon, M. Geha, B. Willman, M.G. Walker, Nature 454, 1096 (2008)

44. M.G. Walker, M. Mateo, E.W. Olszewski, O.Y. Gnedin, X. Wang, B. Sen, M. Woodroofe, Ap. J. 667, L53 (2007)

45. J. Barnes, G. Efstathiou, Ap. J. 319, 575 (1987)

46. S. Gottlöber, G. Yepes, Ap. J. 664, 117 (2007)

47. B.W. O'Shea, M.L. Norman, Ap. J. 654, 66 (2007)

48. R.S. de Souza, B. Ciardi, U. Maio, A. Ferrara, Mon. Not. R. Astron. Soc. 428, 2109 (2013)

49. F.S. Guzmán, F.D. Lora-Clavijo, J.J. González-Avilés, F.J. Rivera-Paleo, arXiv:1310.3909

50. M.P. Silverman, R.L. Mallet, Gen.Rel.Grav. 34, 633 (2002)

51. R.P. Yu, M.J. Morgan, Class.Quant.Grav. 19, L157 (2002)

52. B. Kain, Y. Ling, Phys.Rev. D 82, 064042 (2010)

53. J.A. González, F.S. Guzmán, Phys.Rev. D 83, 103513 (2011)

54. D. Castañeda Valle, E.W. Mielke, Ann.of Phys. 336, 245 (2013)

55. T.-P. Woo, T. Chiueh, Ap. J. 697, 850 (2009) 